\title{
Rastreo, prevención y tratamiento de las complicaciones de la diabetes tipo II: de la Evidencia a la práctica clínica
}

Screeninig, prevention, counseling, and treatment for the complications of type 2 diabetes mellitus. Putting evidence into practice. Vijan S, Stevens DL, Herman WH, Funnell MM, Standiford CJ. J Gen Intern Med 1997;12:567-80.

\begin{abstract}
Introducción
Esta guía resume la evidencia disponible para el manejo (detección, prevención y tratamiento) de las complicaciones de la diabetes (DBT) tipo II. Los estudios clínicos revisados fueron identificados en el MEDLINE y analizados por un grupo multidisciplinario integrado por personal que atiende pacientes diabéticos. La evidencia ha sido integrada en la presente guía.

Debido a que el manejo de pacientes diabéticos es un proceso complejo, la guía pretende ayudar a los médicos a incorporar la prevención y el tratamiento precoz de las complicaciones en su práctica.
\end{abstract}

\section{La DBT y sus complicaciones son un problema de salud pública} La DBT tipo II es una condición prevalente que afecta del 2 al 3\% de los adultos. Clásicamente se manifiesta luego de los 30 años y en pacientes con más del 120\% del peso ideal. Está asociada a una importante morbilidad. La prevención y tratamiento de las complicaciones puede potencialmente mejorar la calidad y la expectativa de vida.

Las enfermedades cardiovasculares son más prevalentes en los pacientes diabéticos, esto conlleva a una mayor mortalidad que la población general. Las complicaciones microvasculares (retino, nefro y neuropatía) pueden evolucionar a estadíos terminales y provocar ceguera, insuficiencia renal crónica (IRC) y amputación.

El rastreo y tratamiento de las complicaciones en estadíos tempranos ha demostrado evitar la evolución hacia estadíos terminales. A pesar de esta evidencia, la implementación del rastreo es baja.

\section{Prevención y tratamiento de las complicaciones macrovasculares Uso de aspirina (AAS) profiláctica \\ Un metaanálisis y un largo estudio randomizado controlado (ERC) han demostrado que los pacientes diabéticos reciben de la AAS la misma pro- tección cardiovascular que los no diabéticos sin mayores efectos adver- sos. Si bien no todos los puntos finales estudiados alcanzaron significa- ción estadística, hay una tendencia a la reducción de eventos vasculares y mortalidad en los pacientes tratados. Los autores recomiendan el uso de AAS profiláctica en pacientes con DBT mayores de 50 años o con otros factores de riesgo asociados de no existir contraindicaciones. La dosis recomendada oscila entre 81 y $325 \mathrm{mg}$ por día.}

\section{Hipertensión arterial (HTA)}

La HTA contribuye al desarrollo de la enfermedad arterioesclerótica (EA) en los diabéticos. La HTA no tratada puede, además, empeorar la nefropatía preexistente. El rastreo y tratamiento de la HTA son componentes fundamentales en el cuidado de los diabéticos.

Los pacientes con DBT desarrollan HTA con el doble de frecuencia que los no diabéticos. El riesgo aumenta con la edad lo que explica que luego de los 45 años el $60 \%$ de los diabéticos tienen HTA. La mayoría tienen HTA esencial o como consecuencia de la nefropatía.

Estudios controlados, no randomizados identificaron el nivel por encima del cual debe iniciarse tratamiento para la HTA en diabéticos tipo II. Los estudios realizados en la población general sostienen el iniciar tratamiento con niveles mayores a 140/90. Los ERC que evaluaron la eficacia del tratamiento antihipertensivo habitualmente excluyeron a los pacientes diabéticos o no han podido analizarlos separadamente. Sin embargo, el Hypertension Detection and Follow up Program coperative Group demostró que en los diabéticos se reduce la mortalidad en un $25 \%$ (cifras relativas similares al resto de los pacientes).

Un subanálisis del Systolic Hypertension in the Eldery Program demostró un 55\% de reducción en la enfermedad coronaria (EC) en diabéticos tratados con bajas dosis de diuréticos.

Si bien el intervalo para controlar la TA no se ha establecido en ERC, los autores recomiendan tomar la TA en cada visita. Si se encuentran varias tomas superiores a 140/90, debe iniciarse intervención. En pacientes con HTA leve, deben recomendarse medidas no farmacológi- cas (reducción de peso, de ingesta de sodio, de alcohol). Los pacientes que no respondan o pacientes con HTA moderada o severa, deben recibir tratamiento farmacológico. Carecemos de evidencia para recomendar la meta a alcanzar en el tratamiento. La recomendación se basa en el Sixty Report of the Join National Comitee on Detection, evaluation and Treatment of High Blood Presure que recomienda llegar a una meta menor a 130/85. Esta meta se basa en marcado riesgo cardiovascular de los pacientes diabéticos. El tratamiento agresivo de la HTA sería beneficioso en pacientes con nefropatía.

Los inhibidores de la enzima convertidora (IECA) son una razonable opción de primera línea debido a los efectos nefroprotectores y la neutralidad sobre el metabolismo (no afectan la glucemia). Los antagonistas de los receptores de angiotensina 2 son buenos antihipertensivos, no causan tos y se está estudiando si tienen el mismo efecto sobre el riñón que los IECA.

Los diuréticos en bajas dosis no parecen tener efectos adversos de importancia y han demostrado disminuir la mortalidad en pacientes con DBT. Las altas dosis de tiazidas aumentan la glucemia y los lípidos y pueden causar impotencia.

El empleo de otros antihipertensivos deben basarse en necesidades individuales del paciente. Los bloqueantes cálcicos (BC) son eficaces en bajar la TA, pero no tienen el mismo efecto sobre el riñón que los IECA. Los betabloqueantes tienen un rol fundamental luego de un infarto agudo de miocardio (IAM). Los diabéticos tienen mayor mortalidad luego de un IAM que los no diabéticos por lo que experimentan mayor cardioprotección que los no diabéticos. Este importante beneficio debe ser tenido en cuenta frente a la posibilidad de que un betabloqueante enmascare síntomas de hipoglucemia. Los bloqueantes de los receptores a adrenérgicos son eficaces en bajar la TA, no tienen efectos metabólicos, pero pueden empeorar la hipotensión postural en pacientes con DBT.

Los estudios muestran que entre un 20 a un $60 \%$ de los pacientes no se controlan con monoterapia. En esos casos la asociación de IECA con diuréticos es razonable. La asociación de diuréticos con betabloqueantes puede empeorar el control metabólico y debe usarse con precaución.

\section{Lípidos}

Los pacientes con DBT tipo II tienen elevados los triglicéridos y las VLDL con bajos niveles de HDL. La hiperlipemia contribuye significativamente al desarrollo de la enfermedad vascular. No se ha realizado ningún ECR para Identificar la meta de tratamiento de la hiperlipemia en diabéticos. Tampoco se han determinado los intervalos entre rastreo. En población no diabética con alto riesgo vascular, ECR demostraron que bajar la LDL disminuye la mortalidad cardiovascular. Debido a la alta prevalencia de enfermedad coronaria en los diabéticos, el National Cholesterol Education Panel (NCEP) recomienda que los diabéticos sean rastreados anualmente como los pacientes con enfermedad coronaria e iniciar tratamiento con LDL mayor de $130 \mathrm{mg} / \mathrm{dl}$ con una meta de LDL menor a $100 \mathrm{mg} / \mathrm{dl}$.

Los inhibidores de la HMG Co A reductasa son efectivos en disminuir la LDL, los beneficios a largo plazo no se han evaluado en diabéticos. Los datos provenientes de estudios son limitados, pero el rastreo y tratamiento de la hiperlipemia en diabéticos podría disminuir la morbimortalidad cardiovascular. Debido a la alta prevalencia de enfermedad coronaria en diabéticos, recomiendan tratar a los diabéticos acorde a la guía del NCEP. La intervención inicial debe ser dieta, luego un inhibidor de la HMG Co A reductasa. El ácido nicotínico debe usarse con precaución por el riego de hiperglucemia.

Los triglicéridos son un factor de riesgo independiente para el desarrollo de enfermadad arterioesclerótica en diabéticos. El tratamiento inicial debe estar dirigido a mejorar el control glucémico. Si con la normalización de la glucemia no se corrige la hipertrigliceridemia, debe considerarse el uso de gemfibrozil sobre todo si la trigliceridemia es $>400 \mathrm{mg} / \mathrm{dl}$. 
El gemfibrozil ha demostrado disminuir la mortatidad cardiovascular, pero no la global en la población general. El análisis de subgrupos muestra que los diabéticos podrían recibir un mayor beneficio del tratamiento de la hipertrigliceridemia a pesar del bajo número de pacientes estudiados. Hasta disponer de mejor evidencia en diabéticos, se recomienda dieta, control glucémico seguido de gemfibrozil (de ser necesario) para el manejo de la hipertrigliceridemia.

\section{Tabaquismo}

Estudios de cohorte longitudinales han mostrado que la DBT y el tabaquismo son factores de riesgo sinérgicos para el desarrollo de enfermedad vascular. Los pacientes deben ser fuertemente aconsejados para abantónar este hábito. Pueden recibir de ser necesario sustitutos de nicotina (chicles o parches). Esto último puede indicarse aún con enfermedad cardiovascular conocida.

\section{Resumen}

A continuación se enumeran diferentes intervenciones y el grado de recomendación. Si al lado de la intervención figura (a) significa que los estudios se han realizado en población general; si figura (b) significa que hay estudios en diabéticos.

\begin{tabular}{llc}
\hline Intervención & Evidencia & Población \\
\hline Tratar con TA mayor de $140 / 90$ & Estudios randomizados y controlados & (a) \\
\hline Meta del tratamiento HTA: $<130 / 85$ & Opinión de panel de expertos & (b) \\
\hline Tratamiento con LDL $>130 \mathrm{mg} / \mathrm{dl}$ & Opinión de panel de expertos & (b) \\
\hline Tratamiento de trigliceridos $>400 \mathrm{mg} / \mathrm{dl}$ Estudios randomizados y controlados & (b) \\
\hline Recomendación para dejar de fumar & Estudios controlados no randomizados & (b) \\
\hline
\end{tabular}

Prevención y tratamiento de las complicaciones microvasculares La diabetes es causa subyacente de nuevos casos de ceguera, IRC y amputación no traumática en los EE-UU. Estas situaciones son el resultado de la progresión de la microangiopatía diabética (retinopatía, nefropatía y neuropatía). El riesgo de desarrollar estas complicaciones puede ser reducido con intervenciones que previenen el desarrollo o enlentecen la progresión de la enfermedad microvascular temprana.

\section{Retinopatía}

En diabetes tipo I, ECR han demostrado que el mejoramiento del control de la glucemia puede disminuir la incidencia de retinopatía. Estudios observacionales en diabéticos tipo II sugieren una relación similar entre el control de la glucemia y la tasa de retinopatía. De modo que la prevención primaria de la retinopatía diabética consiste en optimizar el control de la glucemia. Además el control metabólico retarda la progresión de la retinopatía una vez establecida. La progresión de la enfermedad ocular a retinopatía proliferativa y edema macular puede causar grave pérdida de la agudeza visual. ECR han demostrado que la terapia con láser de la retinopatía proliferativa y del edema macular reduce significativamente la pérdida visual. A pesar de esto, sólo al 35-60\% de los pacientes diabéticos se les realiza fondo de ojo anualmente. Si bien podría recomendarse fondo de ojo menos frecuentemente en pacientes con bajo riesgo de retinopatía (fondo de ojo basal normal, excelente control glucémico), no se ha realizado una estratificación formal del riesgo que permita hacer una recomendación en este sentido.

Los estudios que han evaluado la sensibilidad y especificidad del fondo de ojo de los médicos de atención primaria, muestran que los médicos entrenados detectan la retinopatía proliferativa, pero fallan en diagnosticar el edema macular. Si bien el intervalo entre rastreos no ha sido evaluado en ECR, se sugiere la evaluación anual de la retina y eventual tratamiento con láser por un especialista.

\section{Nefropatía}

El óptimo control de la glucemia actúa como prevención primaria de
La nefropatía diabética.

El control agresivo de la hipertensión arterial y los inhibidores de la enzima convertidora juegan un rol fundamental en la prevención de la enfermedad renal.

La microalbuminuria y la proteinuria han sido identificadas como signos tempranos de nefropatía diabética. Si bien el intervalo entre rastreos óptimo no ha sido determinado, el rastreo permite realizar intervenciones que claramente reducen la progresión a IRC.

El esquema propuesto para el rastreo de nefropatía diabética es el siguiente:

a) Realizar una tira reactiva en orina anualmente para detectar proteinuria franca, hemoglobinuria, etc.

b) Si la proteinuria es negativa, realizar determinación de microalbuminuria y confirmarla de ser positiva.

c) Si se confirma la microalbuminuria: chequear creatinina y electrolitos, comenzar con un inhibidor de la enzima convertidora y realizar control agresivo de la hipertensión (estas dos últimas recomendaciones provienen de evidencias grado 1 de estudios realizados en diabéticos).

d) Si al realizar la tira reactiva se detecta proteinuria franca, ésta debe confirmarse y debe procederse como en el punto $c$ ).

El diagnóstico de nefropatía diabética requiere la presencia de albuminuria en un paciente con diabetes de más de 5 años de evolución o con evidencia de retinopatía diabética. Dado que existen otras causas de nefropatía con proteinuria, las personas que no reúnan los criterios arriba mencionados o tengan rasgos sugestivos de otras enfermedades renales (sedimento urinario activo, proteinuria de rango nefrótico, hipertensión acelerada o insuficiencia renal rápidamente progresiva) requerirán mayor evaluación.

El control de la presión arterial y los inhibidores de la enzima convertidora han demostrado disminuir la progresión de la nefropatía en forma independiente en trabajos realizados con diabéticos tipo II. Otros antihipertensivos pueden disminuir la progresión de la nefropatía pero por su efecto antihipertensivo únicamente, es decir no tienen efecto independiente sobre la función renal. Los inhibidores de la enzima convertidora deben usarse como antihipertensivos de primera línea en diabéticos con nefropatía a menos que existan contraindicaciones. El control estricto de la presión es de suma importancia. Los niveles de tensión arterial que debemos fijarnos como meta en diabéticos con nefropatía no han sido fehacientemente establecidos, sin embargo hay clara asociación entre la presión arterial y la progresión de la nefropatía. En pacientes normotensos con microalbuminuria, la dosis de inhibidores de la enzima de conversión no está claramente definida. Se recomienda titular la dosis hasta dosis máxima o aparición de efectos colaterales. La restricción proteica de la dieta no ha demostrado ser efectiva en la prevención de la progresión de la nefropatía.

\section{Nefropatía y cuidado de los pies}

Los pacientes diabéticos tienen mayor riesgo de desarrollar neuropatía periférica y úlceras en los pies. La neuropatía suele estar presente en el $50 \%$ de los diabéticos con más de 15 años de evolución. La optimización de la glucemia reduce la incidencia de neuropatía en los diabéticos tipo I. Trabajos epidemiológicos sugieren esta misma asociación en los tipo II. La evidencia sugiere que la detección temprana de la neuropatía diabética disminuye el número de internaciones, las úlceras y las amputaciones.

Debe realizarse un test para evaluar la sensibilidad con un monofilamento de nylon periódicamente (podría ser anualmente). De no poseer monofilamento el diapasón debe utilizarse para evaluar palestesia. La combinación de educación al paciente en el cuidado de los pies y el control de los factores de riesgo para amputaciones se han evaluado en un estudio randomizado y en una cohorte. Se ha demostrado una reducción significativa de lesiones serias en los pies.

Se recomienda en cada visita regular inspeccionar los pies de los dia- 
béticos, identificar callos, deformidades y anomalías estructurales. Los pacientes con examen del pie anormal deben recibir educación acerca del cuidado que deben dar a sus pies.

Hay drogas en investigación para el tratamiento de la neuropatía diabética (por ejemplo los inhibidores de la aldolasa reductasa).

El dolor de la neuropatía diabética puede tratarse con antidepresivos tricíclicos en dosis bajas.

Los estudios muestran que los pacientes con úlceras en los pies tienen mejores resultados si son tratados por un equipo multidisciplinario especializado en pie diabético.

\section{Resumen}

A continuación se enumeran diferentes intervenciones para prevención de la microangiopatía y el grado de recomendación. Si al lado de la intervención figura (a) significa que los estudios se han realizado en población general; si figura (b) significa que hay estudios en diabéticos.

\begin{tabular}{|c|c|c|}
\hline Intervención & Evidencia & Población \\
\hline Tratamiento de la retinopatía con láser & Estudios randomizados y controlados & (b) \\
\hline Considerar mejorar el control glucémico & Estudios randomizados y controlados & (b) \\
\hline Control agresivo de la HTA & Estudios observacionales & (b) \\
\hline $\begin{array}{l}\text { IECA en presencia de microalbuminuria } \\
\text { o proteinuria }\end{array}$ & Estudios randomizados y controlados & (b) \\
\hline Control agresivo de La HTA & Estudios randomizados y controlados & (b) \\
\hline $\begin{array}{l}\text { Optimizar el control glucémico ante } \\
\text { evidencia de neuropatía }\end{array}$ & Estudios randomizados y controlados & (b) \\
\hline $\begin{array}{l}\text { Derivación para atención en equipo } \\
\text { multidisciplinario de existir úlcera en pie }\end{array}$ & Estudios randomizados y controlados & (b) \\
\hline Dosar Hb glicosilada cada 6 meses & Estudios randomizados y controlados & (b) \\
\hline
\end{tabular}

\section{Control de la glucemia}

El DCCT (un importante estudio randomizado y controlado realizado en diabéticos tipo I), demostró que el control estricto de la glucemia reduce el desarrollo y la progresión de las complicaciones microvasculares. Un estudio randomizado realizado en Japón con diabéticos tipo II ha confirmado que la tasa de complicaciones microvasculares puede reducirse si se mejora el control de la glucemia medido por los niveles de hemoglobina glicosilada ( $\mathrm{Hb}$ glicosilada).

Es decir que el control estricto de la glucemia parece disminuir la progresión y aparición de microangiopatía si bien en la diabetes tipo 2 la experiencia es limitada.

El efecto del control de la glucemia sobre la enfermedad macrovascular en diabéticos tipo II permanece incierto, si bien hay estudios en marcha para responder esta pregunta. El mayor riesgo del control estricto es la hipoglucemia. En un trabajo en marcha sobre control estricto en diabéticos tipo II este efecto adverso se observó en menos del $2 \%$ por. año.

La hemoglobina glicosilada es una buena medida del control metabólico en el largo plazo. Este indicador debería ser medido cada 6 meses en pacientes estables y cada 1 a 3 meses si se realizan modificaciones en el tratamiento. Esta recomendación se basa en la vida media de la hemoglobina glicosilada.

La meta a alcanzar de hemoglobina glicosilada debe acordarse entre el paciente y el médico en la primera visita. Existen diversos factores a considerar para fijar la meta. En pacientes jóvenes y por lo demás saludables, el riesgo de desarrollo de complicaciones crónicas es una preocupación. En estos pacientes el control estricto de glucemia debe ser una meta. En situaciones en las cuales existen factores que puedan afectar la ecuación riesgo/beneficio en el control estricto de la glucemia, la meta es menos clara. En las siguientes situaciones el beneficio del control estricto puede verse limitado: Insuficiencia cardíaca congestiva, cáncer avanzado, complicaciones por la diabetes avanzadas (insuficiencia renal terminal), incapacidad para llevar a cabo el tratamiento, limitada expectativa de vida. En ciertas circunstancias el riesgo de hipoglucemia por control estricto supera los potenciales beneficios de dicho control: his- toria de hipoglucemia severa, enfermedad cardiovascular o cerebrovascular avanzada, neuropatía autonómica (especialmente cardíaca), movilidad reducida o persona que vive sola.

\section{Manejo de la glucemia}

El plan alimentario y el ejercicio constituyen los pilares del manejo de la hiperglucemia. Los pacientes que no logran un adecuado control con estas medidas son candidatos a recibir medicación. Las opciones farmacológicas incluyen sulfonilureas, biguanidas (metformina, inhibidores de la alfa glucosidasa intestinal (acarbosa), tiazolidinedionas (troglitazona) e insulina subcutánea. Si se requiere tratamiento farmacológico, igualmente debe asociarse al plan alimentario y a la actividad física.

\section{Plan alimentario}

El plan alimentario constituye un pilar en el tratamiento. También es la primera línea de manejo. A pesar de que la reducción de peso ha sido tradicionalmente el foco del tratamiento alimentario de los diabéticos tipo II, las estrategias actuales en materia de dieta así como las dietas muy hipocalóricas han demostrado ser ineficaces en lograr reducción del peso a largo plazo. Por lo tanto se recomienda que la terapia nutricional se dirija a lograr las metas de glucemia, lípidos y tensión arterial.

No existe una única "dieta para diabéticos". Se recomienda la interconsulta con personal idóneo en la confección de planes alimentarios para diabéticos.

Una moderada pérdida de peso reduce la hiperglucemia, la dislipidemia, la hiperetensión aunque el peso ideal no sea alcanzado. Es preferible enfatizar en un peso razonable más que en el peso ideal. Definimos peso razonable como aquél peso que tanto el médico como el paciente consideran que puede lograrse mantenerse en el tiempo. Para muchas personas, aun aquéllas con sobrepeso, este peso razonable puede ser su peso actual o 5 a $10 \mathrm{~kg}$ menos.

\section{Sulfonilureas}

Las sulfonilureas han sido empleadas como primera línea de tratamiento en diabéticos tipo II que requieren fármacos. Actualmente se prefieren aquellas de segunda generación y vida media más corta. Actúan primariamente por aumento de la secreción de insulina pancreática, podrían también aumentar la sensibilidad del receptor. Los pacientes pueden tratarse inicialmente con baja dosis y aumentarla gradualmente hasta alcanzar el control o llegar a dosis máxima. Si con dosis máximas no se lograra el control, puede asociarse una biguanida, acarbosa o insulina.

\section{Biguanidas}

La metformina puede ser seleccionada como droga de primera línea en el tratamiento de la diabetes tipo II cuando el tratamiento no farmacológico no resulta suficiente. La metformina disminuye la glucemia a través del aumento de la sensibilidad periférica a la insulina y de la disminución de la producción hepática de glucosa. Debe evitarse en los pacientes con insuficiencia cardíaca o renal o hepática. Puede observarse anorexia, náuseas, diarrea o dolor abdominal hasta en el $30 \%$ de los pacientes. La dosis inicial es de $500 \mathrm{mg}$ por día y se puede aumentra de a $500 \mathrm{mg}$ por semana hasta un máximo de $2.5 \mathrm{~g}$ por día. Si no se logra el control con dosis máximas, debe considerarse la combinación de drogas.

\section{Inhibidores de la alfa glucosidasa}

Los inhibidores de la alfa glucosidasa como la acarbosa pueden utilizarse como monoterapia en conjunto con la dieta. La acarbosa disminuye la digestión de los hidratos de carbono, retarda la absorción de glucosa y disminuye la glucemia posprandial. La dosis inicial es de $25 \mathrm{mg}$ tres veces por día y debe tomarse con el primer bocado de cada comida principal. Los efectos adversos incluyen dolor abdominal, meteorismo y diarrea. Son muy comunes, pero disminuyen con el uso en 4 a 8 semanas. La dosis de mantenimiento es de 50 a 100 mg tres veces por día. 


\section{Tiazolidenionas}

Este es una nueva clase de agentes orales. La troglitazona es la primera droga de esta clase. Puede usarse para el tratamiento diabeticos tipo II que reciben insulina y no se controlan adecuadamente.

\section{Terapia con sulfonilureas más insulina al acostarse}

Este esquema debe considerarse en pacientes que no se controlan con dosis máximas de hipoglucemiantes. Deben prescribirse sulfonilureas a dosis máximas e insulina NPH al acostarse en una dosis de $0.3 \mathrm{U} / \mathrm{kg}$ de peso y ajustarla según resultados. En un estudio se requirió una dosis promedio de $64 \mathrm{U}$ para lograr el control glucémico.

\section{Insulina}

Si el tratamiento combinado fracasa, debe indicarse insulina según necesidades (inyección diaria, 2 inyecciones diarias, múltiples dosis, etc).

\section{Educación para el autocuidado}

En el momento del diagnóstico, los pacientes deben recibir información acerca de la enfermedad y su manejo incluyendo la importancia del autocontrol.

Meta-análisis y revisiones han demostrado que la educación mejora lo que el paciente conoce de la enfermedad, el autocuidado, la situación psicosocial y el control metabólico.

Un estudio epidemiológico realizado en Italia ha demostrado que la limitación al acceso al cuidado y la falta de educación diabetológica constituyen factores de riesgo para complicaciones. Otros estudios han demostrado que la educación para el cuidado de los pies reduce la tasa de amputaciones.

En la siguiente tabla pasamos lista a los tópicos y preguntas de autocuidado que deben considerarse.

\section{Tópicos de autocuidado}

\section{En cada visita regular preguntar por:}

* Responsabilidad activa: ¿Toma Ud. responsabilidad en el cuidado diario por su DBT?

* Meta de glucemia: ¿Conoce Ud. el último valor de Hb glicosilada y cuán lejos o no está de la meta?

* Monitoreo diario en pacientes con insulina: ¿Conoce Ud. La racionalidad del monitoreo? ¿Cuál es su agenda? ¿Cómo están los resultados? ¿Y cómo los utiliza?

* Medicación: ¿Cómo la toma/se inyecta? ¿Las toma aunque esté enfermo y no pueda comer? ¿Qué dosis recibe?

* Ejercicio: ¿Qué ejercicio realiza para mantener la glucemia normal?

* Plan alimentario: ¿Puede Ud. realizar su plan alimentario?

* Preguntas sin respuesta: ¿Tiene Ud. dudas no aclaradas? ¿Le gustaría consultar con la dietista/enfermera?

* ¿Tiene alguna pregunta que me quiera formular?

\section{Al menos anualmente, preguntar por:}

* Situaciones de emergencia: ¿Cuáles son los síntomas y el tratamiento de la hipoglucemia? ¿Y de la hiperglucemia?

¿Cuándo debe consultar con el médico?

* Identificación: ¿Lleva Ud. identificación de diabético?

* Rastreo de complicaciones: ¿Conoce Ud. el resultado del rastreo de complicaciones? ¿Cuándo debe ser rastreado

nuevamente?

* Cuidado de pies: ¿Qué realiza Ud. para cuidar sus pies? ¿Mira sus pies todos los días?

* Sitios de inyección: ¿Rota los sitios de inyección? ¿Los inspecciona?

\section{Sugerencia de la frecuencia para realizar intervenciones en diabéticos (basadas en opinión de expertos)}

En cada visita regular
*Control de TA
*Inspección de pies
*Peso
* Reforzar medidas de autocuidado(ver tabla)
*Monitoreo
* Régimen farmacológico
*Plan alimentario
*Planificación familiar
*Situación psicosocial

\section{Cada 3 a 6 meses Anualmente}

*Hemoglobina glicosilada *Status fumador

* Medición de lípidos

* Fondo de ojo por el especialista

* Proteinuria, si es negativa, microalbuminuria

*Test del monofilamento (o diapasón)

* Revisar medidas de autocuidado (tabla): hipoglucemia, hiperglucemia, tarjeta identificadora, rastreo de complicaciones, cuidado del pie, monitoreo de sitios de inyección (insulina)

\section{COMENTARIO}

Los paneles de expertos han publicado guías acerca del manejo apropiado de los pacientes con DBT tipo II. Habitualmente han sido basadas en revisiones de la literatura o expertos en el área, pero no han sido "basadas en la evidencia". Es decir no han incorporado un método explícito para la revisión bibliográfica.

Esta guía ha sido confecionada en base a criterios estrictos de selección de los trabajos.

\section{Dra. Karin Kopitowski}

Unidad de Medicina Familiar y Preventiva. Hospital Italiano de Buenos Aires.
Muchas preguntas aún no tienen respuesta. Está en marcha un gran estudio prospectivo (United Kingdom Prospective Diabetes Study y The Heart Outcomes Prevention Evaluation) que ayudará a responderlas. Cabe destacar que desde la publicación de esta guía han sido publicados estudios randomizados controlados que también avalan el uso de troglitazona como monoterapia inicial, aunque con pocos pacientes (1-2).

\section{Referencias}

1. Inzucchi SE, Maggs DG, Spollett GR et al. Efficacy and Metabolic Effects of Metformin and Troglitazone in Type II Diabetes Mellitus. N EngL J Med 1998;338:867-72

2. Schwartz S, Raskin P, Fonseca V et al for the Troglitazone and Exogenous Insulin Study Group. Effect of Troglitazone in Insulin-Treated Patients with Type II Diabetes Mellitus. N Engl J Med 1998:338:861-6 